\title{
Article@Virology
}

\section{Resveratrol Inhibits High Glucose-induced Activation and Cytokine Production of Isolated Primary Pancreatic Stellate Cells}

Zhen Zhou ${ }^{1} \Delta$, Xiaodong $\mathrm{Sun}^{1} \Delta$, Yan Rao ${ }^{2}$, Jinfeng $\mathrm{An}^{3}$, Xinjian Zhou ${ }^{1}$, Mingyi Li ${ }^{1}$, Xinsheng $\mathrm{Gu}^{1}$, Xincai $\mathrm{Hao}^{1}$, Ming Sang ${ }^{1 *}$

1. Department of Endocrinology, Xiangyang No.1 People's Hospital, Hubei Key Laboratory of Wudang Local Chinese Medicine Research, Hubei University of Medicine, Xiangyang 441000, P. R. China

2. ABSL-III Laboratory at the Center for Animal Experiment, Wuhan University School of Medicine, Wuhan 430071, P. R. China

3. Xiangyang Anding Hospital, Xiangyang 441000, P. R. China

\section{ABSTRACT}

Objective: Activation of pancreatic stellate cells (PSCs) is detrimental to pancreas function by promoting pancreatic fibrosis. Resveratrol is a natural and pharmacologically active compound. This study is to investigate the effect of resveratrol on the bilogical behavior of PSCs under high glucose condition. Methods: Isolated primary mouse PSCs were cultured in low glucose $(5.5 \mathrm{mmol} / \mathrm{L}$ glucose, LG group) medium, high glucose $(25 \mathrm{mmol} / \mathrm{L}$ glucose, HG group) medium and treated with resveratrol $(25 \mu \mathrm{mol} / \mathrm{L}$ or $50 \mu \mathrm{mol} / \mathrm{L})$. Cell proliferation was examined using MTT assay. The expression of $\alpha$-SMA and collagen I were determined using Western blotting. Alpha-SMA expression was also determined using immuno-cytochemistry staining. IL-1, IL-6, and TNF- $\alpha$ mRNA levels and secretion levels in media of PSCs were determined using qRT-PCR and ELISA respectively. Results: Cell Proliferation, $\alpha$-SMA and collagen I expression levels, IL-1, IL-6, and TNF- $\alpha$ mRNA levels and secretion levels of PSCs were increased after high glucose treatment, compared with low glucose treatment. They were significantly decreased in PSCs treated with both high glucose and resveratrol, compared with high glucose treatment. Conclusion: Resveratrol inhibited high glucose induced PSCs proliferation, activation, cytokine expression and secretion in PSCs. Therefore, resveratrol can be potentially used in therapy of diseases such as type 2 diabetes mellitus, pancreatitis and pancreatic cancer where PSCs is activated by high glucose.

Copyright@2012-2020 Published by Hong Kong Institute of Biologicals Standardization Limited. All rights reserved.

Article history: Submitted: 10/06/2019; Revised: 30/06/2019; Accepted: 03/07/2019

DOI: $10.21092 /$ jav.v8i3.112

Key Words: Type 2 diabetes mellitus; Pancreatic stellate cells; Resveratrol; Islet fibrosis.

$\triangle$ These authors contributed equally to this work.

* Corresponding author, Tel: ,086-710-3420011; E-mail: smxd2000@126.com Copyright@2012-2020 Published by Hongkong Institute of Biologicals Standardization Limited. All rights reserved. 


\section{Introduction}

Pancreatic stellate cells (PSCs) are located between pancreatic lobules and the surrounding area of acinars and contribute to 4-7\% all pancreatic cells under physiological condition. PSCs, characterized by the presence of vitamin A laden lipid droplets, are essential for maintaining the normal pancreatic architecture. Under the pathological conditions, PSCs are activated and can be transformed into myofibroblast -like cell with perinuclear lipid droplets disappear from the cytosol and expression of the activation marker, alpha smooth muscle actin. Activated PSC is the main source of the accumulation of extracellular matrix (ECM) protein, leading to pancreatic fibrosis in chronic pancreatitis and pancreatic cancer [1-4].

T2DM is a disease characterized by hyperglycemia. Studies showed that PSCs were proliferated and activated under hyperglycemia ${ }^{[5]}$. High glucose increased oxidative stress in primary mouse PSCs, thereby facilitating the activation of these cells. This can be attenuated by antioxidant treatment ${ }^{[1]}$. High glucose increases extracellular matrix production in pancreatic stellate cells by activating the renin-angiotensin system ${ }^{[6]}$. High glucose activates mouse pancreatic stellate cells through protein kinase $\mathrm{C}$ and p38 mitogen-activated protein kinase pathway ${ }^{[7]}$. High glucose aggravates the detrimental effects of pancreatic stellate cells on beta-cell function in $\mathrm{T}_{2} \mathrm{DM}^{[8,9]}$.

Resveratrol is a natural polyphenol. It has been reported with a variety of beneficial effects such as antioxidant, anti-aging and anti-inflammatory bioacti-vities; thus often utilized as an active substance in human and veterinary therapeutics ${ }^{[10-13]}$. Recent studies has shown that Resveratrol Inhibits ROS-Promoted Activation and Glycolysis of Pancreatic Stellate Cells via Suppression of miR-21 ${ }^{[14]}$. trans-resveratrol may serve as a therapeutic or an adjuvant agent in anti-fibrotic approaches and/or PSC-relating pathologies $^{[15]}$. The effects of resveratrol on the biological properties of PSCs deserve further investigation.

In this study, we investigate the effects of resveratrol on proliferation, activation, cytokine expression and secretion of isolated primary PSCs treated with high glucose. Our finding provided a clue to use resveratrol in therapy of diseases such as T2DM, pancreatitis and pancreatic cancer where PSCs is activated by high glucose.

\section{Materials and methods}
1.1 Isolation, culture, and verification of primary mouse PSCs

Isolation, culture, and verification of mouse PSCs was performed according to the published method ${ }^{[16,17]}$ with modifi-cation. Briefly, adult male C57BL/6 
mouse (14 weeks old) was anaesthetized with ether and sacrificed by. The mouse was then executed by cervical vertebra dislocation. After soaking in $75 \%$ ethanol for 3 minutes, the mouse was dissected and the pancreas was collected. The pancreatic tissue was cut into $0.5 \mathrm{~mm} 3$ in size and digested using $5 \mathrm{ml}$ of $2.5 \mathrm{~g} / \mathrm{L}$ trypsin (Sigma-Aldrich) and $\quad 0.001 \% \quad$ DNase (Sigma-Aldrich) for $10 \mathrm{~min}$. F-12/DMEM with FBS was added and the digestion was centrifuged at $100 \mathrm{~g}$ for $20 \mathrm{~min}$. After supernatant was discarded, the precipitate was resuspended in F-12/DMEM medium with $10 \%$ fetal bovine serum, $500 \mathrm{u} / 1$ penicillin, and $500 \mathrm{u} / 1$ streptomycin and cultured in a cell culture bottle or dish at $37^{\circ} \mathrm{C}$ with $5 \% \mathrm{CO}_{2}$.

The purity of the isolated cells was examined by morphological observation under a microscope at the second day after isolation. Lipid droplets in PSCs were assessed using Oil Red $\mathrm{O}$ staining and fluorescence excited at $328 \mathrm{~nm}$. Activation of PSCs was evaluated using immunofluo-rescence staining of $\alpha$-SMA after 7 weeks of culture. The cells were routinely sub-cultured every 2-3 days. The 2-4 generation PSCs in the logarithmic growth phase were used in this study.

All experiments related to animal care and all procedures were performed in accordance with relevant guidelines and regulations. This study was approved by the Ethical Committee for Animal Experimenta- -tion of Xiangyang No.1 People's Hospital.

\subsection{MTT assay}

MTT assay was used to determined of cell proliferation of PSCs. PSCs were seeded into 96-well plates at a density 6.0 x103 cells/well, synchronized in medium containing $1 \%$ serum for 12 hours, and then cultured with low glucose medium (5.5 mmol/L glucose, LG) or high glucose medium (25mmol/L glucose, HG) containing resveratrol $(25 \mu \mathrm{mol} / 1$ or $50 \mu \mathrm{mol} / 1)$ or its vehicle control. Each treatment had five replicates. After treatment for $12,24,36$, and $24 \mathrm{~h}$, MTT $(5 \mathrm{mg} / \mathrm{ml})$ was added and incubated for additional $4 \mathrm{~h}$. Supernatant was then discarded and $150 \mu$ l of dimethyl sulfoxide was added to each well. The plate was then read using a microplate reader at $492 \mathrm{~nm}$ wavelength. The experiment was repeated three times.

\subsection{Quantitative reverse transcription- -polymerase chain reaction}

RT-qPCR was used to determined the relative IL-1, IL-6, and TNF- $\alpha$ mRNA levels. After treatment with glucose and resveratrol for $24 \mathrm{~h}$, PSCs were processed using a RNAfast 200 extraction kit and total RNA was extracted. Complementary DNA was synthesized using a TakaRa reverse transcriptase kit. Quantitative RT-PCR reaction was set up using $2 \mu \mathrm{l}$ of cDNA, 
$1 \mu \mathrm{l}$ of gene upstream and downstream primers, $10 \mu \mathrm{l}$ of $2 \mathrm{xSYBY}$ Green PCR Master mix and $6 \mu \mathrm{l}$ of dd $\mathrm{H}_{2} \mathrm{O}$.

The conditions for PCR reaction were as follows: initial denaturation for 10 minutes at $95{ }^{\circ} \mathrm{C}$, followed by 35 cycles of $95{ }^{\circ} \mathrm{C}$ for $30 \mathrm{~s}$ and $60^{\circ} \mathrm{C}$ for $30 \mathrm{~s}$, extension at $74^{\circ} \mathrm{C}$ for $30 \mathrm{~min}$, and final extension at $74{ }^{\circ} \mathrm{C}$ for 10 min. Each treatment group had three replicates. $\beta$-actin was used as the internal reference. The relative mRNA levels of target genes were calculated based on the $2 \Delta \Delta \mathrm{CT}$ method.

The primers were been as follows:

$\beta$-actin up 5'-CTTCCAGCCTTCCTTC CTGG-3' and down 5'-CTG TGTTGGCG TACAGGTCT-3';

IL-1 up 5'-ATAAGCCCACTCTACA CCT-3' and down 5'-ATTGGCCCTGA AAGGAGAGA-3';

IL-6 up 5'-ACGCTAGTCCTCCACG AT-3' and down 5'-GGTTGTTTAACA TTGCCTTT-3';

TNF- $\alpha$ up 5'-GGATCTCAAAGACAA CCAAC-3' and down 5'-ACAGAGCA ATGACTCCAAAG-3'.

\subsection{Western blotting}

Western blotting was used to determined $\alpha$-SMA protein levels. After treatment with glucose and resveratrol for $24 \mathrm{~h}$, PSCs were collected into RIPA cell lysis buffer. A total of $20 \mu \mathrm{g}$ protein for each sample was denatured in loading buffer at $70^{\circ} \mathrm{C}$ for 30 min and resolved by sodium dodecyl sulfate polyacrylamide gel electrophoresis (SDS-
-PAGE). The resolved protein was transferred to a PVDF membrane. The membrane was blocked with $10 \%$ skimmed milk powder in PBST (1:1000 dilution) at room temperature for $2 \mathrm{~h}$ and then incubated with primary antibody (1:5000 dilution) for $4 \mathrm{~h}$ and secondary antibody (1:5000 dilution) for $2 \mathrm{~h}$. The membrane was then washed with PBST and developed using chemiluminescence reagent.

\subsection{Immunoflurescence assay}

Immunoflurescence assay was used to determine $\alpha$-SMA expression in PSCs. PSCs were seeded in 12-well plates at a density of $1 \times 10^{5}$ cells/well and then treated with desired chemicals for $24 \mathrm{~h}$. Naive PSCs and treated PSCs were washed with PBST, fixed with $4 \%$ polyoxymethylene for $15 \mathrm{~min}$, and treated with $0.5 \%$ Triton $\mathrm{X}-100$ (PBS preparation) for $20 \mathrm{~min}$. The cells were blocked using normal goat serum and then incubated with anti- $\alpha$-SMA antibody (1:150 dilution) at $4^{\circ} \mathrm{C}$ overnight.

After wash with PBST, the cells were incubated with secondary antibody (1:500) at room temperature for $1 \mathrm{~h}$ in the dark room thereafter. DAPI was added to the wells after wash with PBST. The cells were examined and recorded under a fluorescence microscope. 


\subsection{ELISA}

ELISA was used to deter-mine IL-1, IL-6, and TNF- $\alpha$ levels in the media of PSCs culture. After treatment with glucose and resveratrol for $24 \mathrm{~h}$, media of PSCs culture were collected. ELISA kit was used to determine IL-1, IL-6, and TNF- $\alpha$ levels in the media of PSCs culture.

\subsection{Reagents}

F-12/DMEM culture medium and fetal bovine serum were purchased from American Gibco Company. Resveratrol and methyl thiazolyl tetrazolium (MTT) was purchased from American Sigma-Aldrich Company. Reverse transcriptase kits and Real-time PCR kit were purchased from Takara Biotechnology (Dalian) Co.Ltd.

Cell total RNA extraction kit (RNAFAST200) was purchased from Shanghai Fastagen Biotechnology Co., Ltd. Protein extraction reagent (RIPA) was purchased from Shanghai Shenneng Gaming Biotechnology Company. Rabbit anti-collagen I and $\alpha$-SMA antibodies were purchased from Wuhan Proteintech Group, Inc. Goat anti-rabbit IgG- HRP conjugate was purchased from Beijing Zhongshan Biolocal Technology Co. Ltd. DyLight 488 fluorophore antibody was purchased from the US Earthox company. ELISA kit was purchased from Shanghai Ren Jie Biotechnology Co., Ltd. The DNA oilgo-nucleotides were designed and synthesized by Shanghai Bioengineering Technology Service Co., Ltd. Resveratrol was dissolved in phosphate-buffered saline (PBS) at the ratio $1 \mathrm{~mol} / \mathrm{L}$ and stored in the refrigerator at $-20^{\circ} \mathrm{C}$.

\subsection{Statistical analysis}

The data measured are expressed using mean $(\bar{x}) \pm$ standard deviation (s) and data was analyzed using SPSS 18.0 statistical software.

Comparisons between groups were performed using single factor variance analysis and LSD-t testing. $\mathrm{P}<0.05$ was considered statistically significant.

\section{Results}

1. Isolation, culture, and verification of primary PSCs

We isolated and cultured PSCs which was spindle- or star-shaped in the first day (resting state PSCs) after isolation (Figure 1A). After cultured for 7 weeks, PSCs were stained with Oil Red $\mathrm{O}$ and immunocytochemistry of $\alpha$-SMA. Oil Red O staining showed that the cytoplasm was rich in lipid droplets (Figure 1B).

The blue-green fluorescence of lipid droplets excited at $328 \mathrm{~nm}$ was visible in cells under fluorescence microscope (Figure 1C). Immunocytochemistry staining showed the expression of $\alpha$-SMA in PSCs (Figure 1D).

These results suggested that the isolated and cultured cells were PSCs and could be activated. 

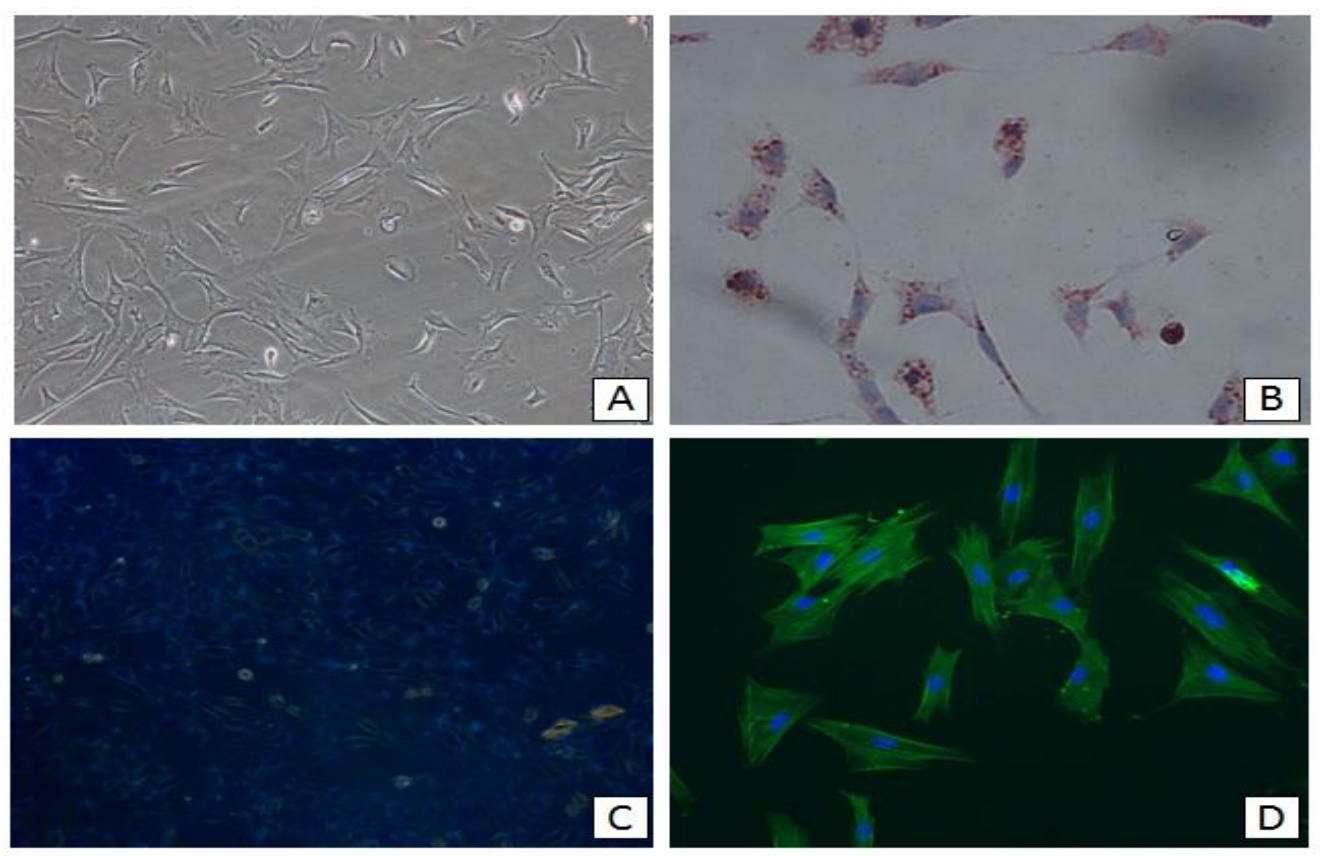

Figure 1: Isolation, culture, and verification of PSCs.

(A) Morphological examination of the cells at the second day after isolation under a phase contrast microscope. The cells were stained with Oil red O (B) and examined for fluorescence of intracellular lipid droplets excited at 328nm (C). After 7 weeks of culture, the cells were stained by immunocytochemistry of $\alpha$-SMA (D). The image showed was taken at $200 \times$.

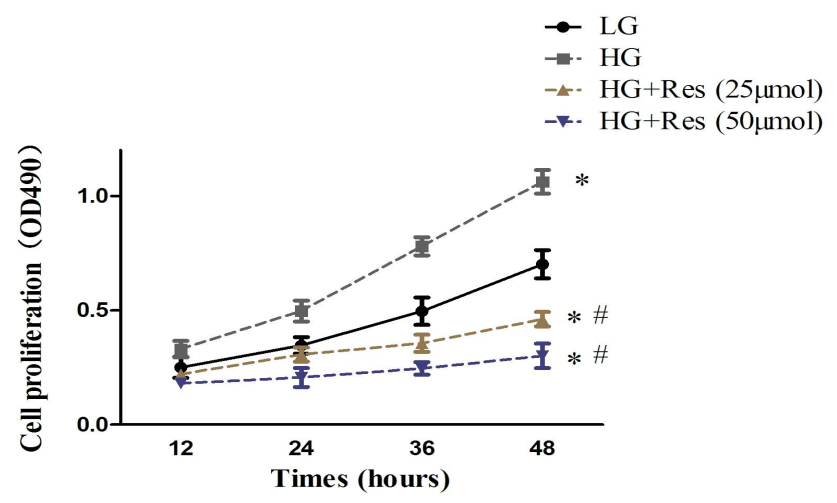

Figure 2: The effect of resveratrol on the proliferation of PSCs in high glucose. PSCs were treated with indicated chemicals. The cell proliferation was determined using MTT assay. * compared with the LG group, $\mathrm{P}<0.05$. \# compared with the HG group, $\mathrm{P}<0.05$. 
2. Resveratrol inhibited high glucose induced PSCs proliferation

To examine the effect of resveratrol on glucose induced PSCs proliferation, we treated PSCs with low high glucose and resveratrol and their respective controls and performed MTT assay. The results showed that proliferation of PSCs was significantly increased after high glucose treatment in a time dependent manner, compared with low glucose treatment (Figure 2). The prolifera-tion of PSCs was significantly decreased after high glucose and resveratrol treatment in a time dependent and resveratrol dose-dependent manner, compared with high glucose treatment and low glucose treatment (Figure 2).

The results suggested that resveratrol inhibited high glucose induced PSCs proliferation.

3. Resveratrol inhibits high glucose-induced PSCs activation

To examine the effect of resveratrol on glucose induced PSCs activation, we treated PSCs with low high glucose and resveratrol and their respective controls for $24 \mathrm{~h}$ and determined expression of Collagen I and $\alpha$-SMA using Western blotting and $\alpha$-SMA expression using immunocytochemistry staining. The results showed that Collagen I and $\alpha$-SMA protein levels in PSCs were significantly increased after high glucose treatment, compared with low glucose treatment (Figure 3).
The Collagen I and $\alpha$-SMA protein levels were significantly decreased after high glucose and resveratrol treatment, compared with high glucose treatment (Figure 3). Similar results of $\alpha$-SMA immunocytochemistry staining were observed in PSCs after treatment with low high glucose and resveratrol and their respective controls (Figure 4).

The results suggested that resveratrol inhibited high glucose induced PSCs activation.

4. Resveratrol inhibited high glucose-induced cytokine expression and secretion in PSCs

To examine the effect of resveratrol on glucose induced cytokine expression in PSCs, we treated PSCs with low high glucose and resveratrol and their respective controls for $24 \mathrm{~h}$ and deter-mined IL-1, IL-6, TNF- $\alpha$ mRNA levels in PSCs cells using quantitative RT-PCR and IL-1, IL-6, TNF- $\alpha$ protein levels in the media of PSCs cells using ELISA. The results showed that IL-1, IL-6, TNF- $\alpha$ mRNA levels in PSCs were significantly increased after high glucose treatment, compared with low glucose treatment (Figure 5). They were significantly decreased after high glucose and resveratrol treatment, compared with high glucose treatment (Figure 5). Similar results of IL-1, IL-6, TNF- $\alpha$ protein levels in the media of PSCs cells were observed 
LG

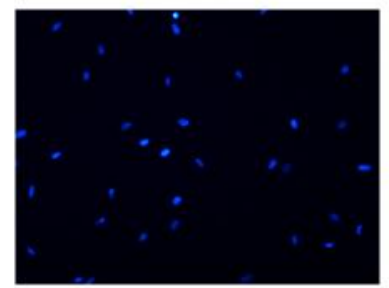

DAPI

$\alpha$-SMA

Merge
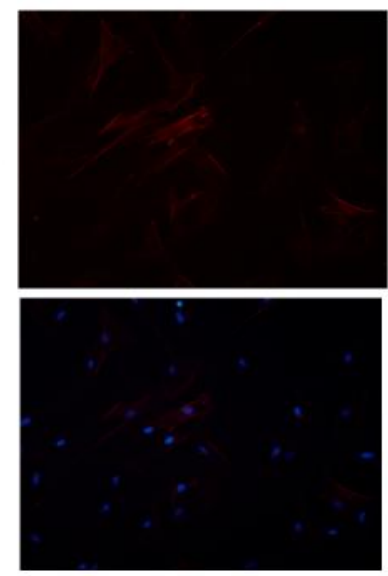

HG
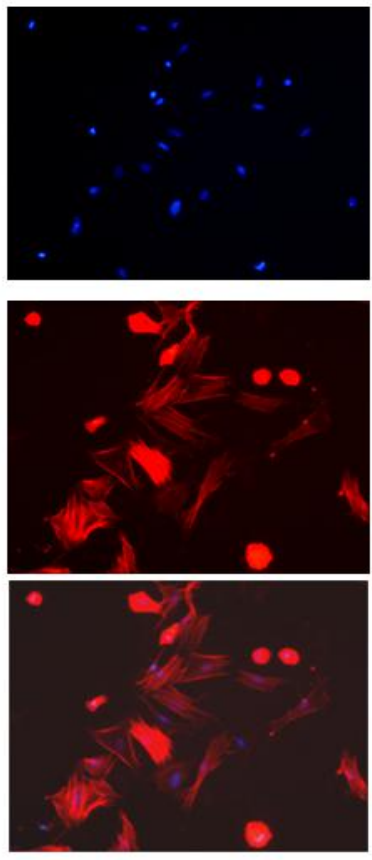

HG+Res
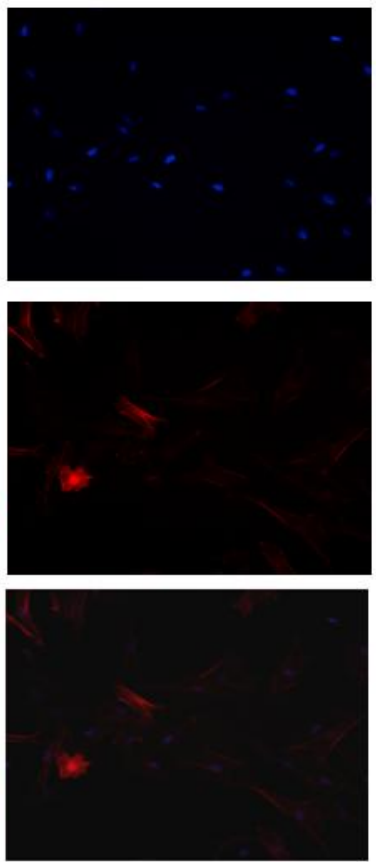

Figure 3. The effect of resveratrol on Collagen I and $\alpha$-SMA levels of PSCs in high glucose.

PSCs were treated with indicated chemicals for $24 \mathrm{~h}$. Collagen I and $\alpha$-SMA levels were determined using Western blotting.

* comparison between two groups, $\mathrm{P}<0.05$.

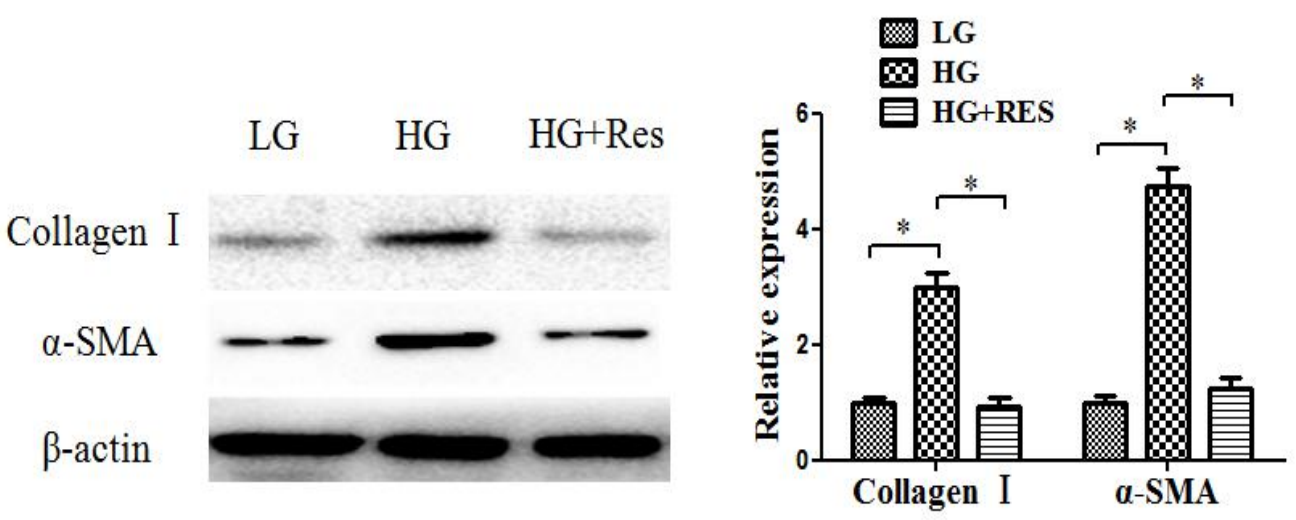

Figure 4. The effect of resveratrol on $\alpha$-SMA levels of PSCs in high glucose. PSCs were treated with indicated chemicals for $24 \mathrm{~h}$. $\alpha$-SMA levels were determined using Immunofluorescence assay.

Copyright@2012-2020 Published by Hongkong Institute of Biologicals Standardization Limited. All rights reserved. 


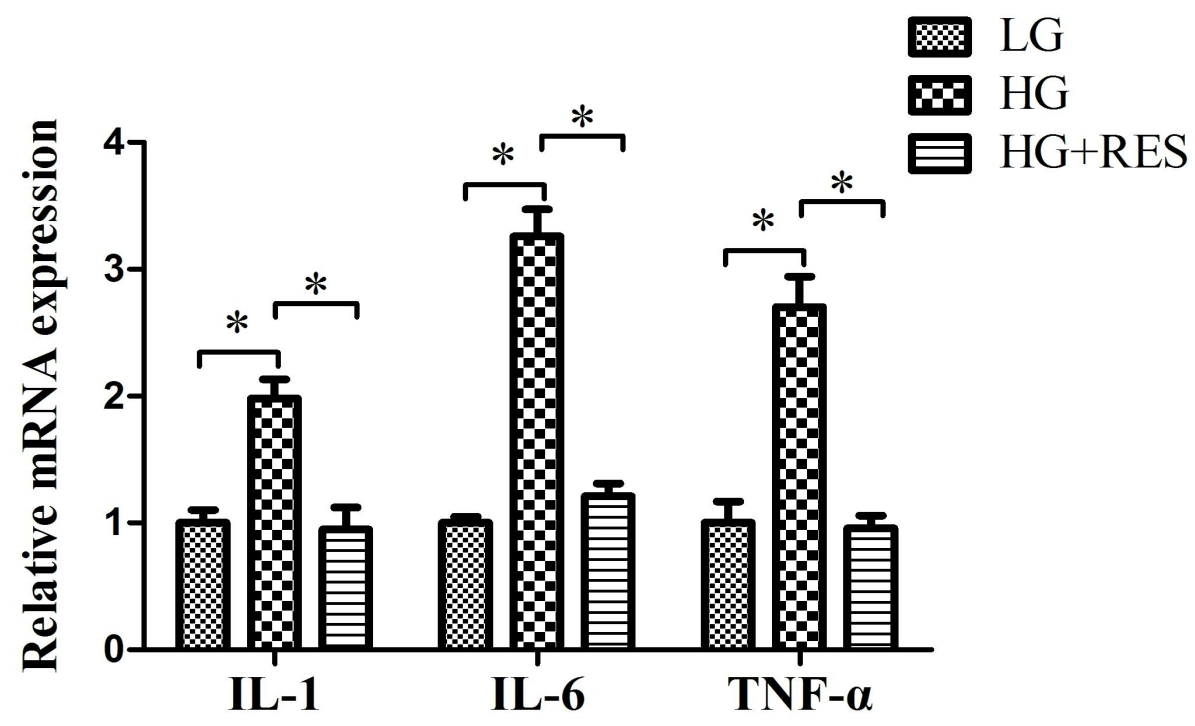

Figure 5. The effect of resveratrol on IL-1, IL-6 and TNF- $\alpha$ mRNA levels of PSCs in high glucose.

PSCs were treated with indicated chemicals for $24 \mathrm{~h}$. IL-1, IL-6 and TNF- $\alpha$ mRNA levels were determined using qRT-PCR.

* comparison between two groups, $\mathrm{P}<0.05$.

in PSCs after treatment with low high glucose and resveratrol and their respective controls (Figure 6). The results suggested that resveratrol inhibited high glucose-induced cytokine expression and secretion in PSCs.

\section{Discussion}

Primary cultures of human and rodent PSCs are good in vitro model to study biological behaviors of PSCs. In order to investigate the effects of resveratrol on the bilogical behavior of PSCs under high glucose condition, we isolated primary PSCs from mouse pancreas based on the published methods ${ }^{[16,17]}$. We found that there were lipid droplets in the cells as examined by Oil Red $\mathrm{O}$ staining and fluorescence, which is a hallmark of resting PSCs. After 7 weeks of culture, the PSCs were activated as indicated by expression of $\alpha$-SMA which is a biomarker of PSC activation. Therefore, we have isolated highly pure PSCs. These PSCs could be activated and used to investigate the effects of resveratrol on their activation . 


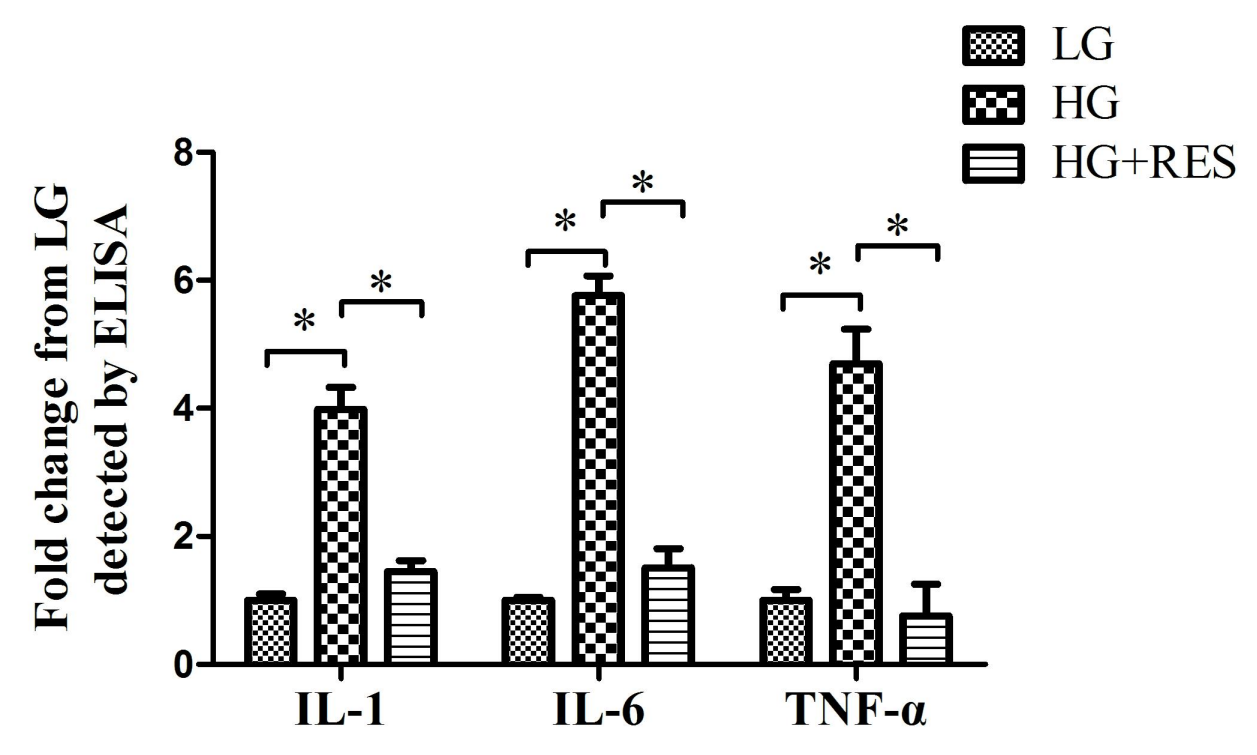

Figure 6: The effect of resveratrol on IL-1, IL-6 and TNF- $\alpha$ protein levels of PSCs in high glucose.

PSCs were treated with indicated chemicals for 24 h. IL-1, IL-6 and TNF- $\alpha$ proteinlevels were determined using ELISA.

* comparison between two groups, $\mathrm{P}<0.05$.

Using isolated primary PSCs, we found that high glucose induced PSCs proliferation and activation. We determined the expression of Collagen I and $\alpha$-SMA to evaluate PSCs activation because Collagen I and $\alpha$-SMA are biomarkers of activation of $\mathrm{PSCs}^{[16,17]}$. These results are consistent with previous studies that showed high glucose induced PSCs activation by increasing oxidative stress $^{[1]}$, activating the renin-angiotensin system ${ }^{[6]}$, through protein kinase $\mathrm{C}$ and p38 mitogen-activated protein kinase pathway ${ }^{[7]}$. We also found that high glucose induced cytokines IL-1, IL-6, Copyright@2012-2020 Published by Hongkong Institute of Biologicals Standardization Limited. All rights reserved.

TNF- $\alpha$ expression and secretion in PSCs. This is consistent with cytokines can be induced in PSCs treated with galectin-3 via integrin signaling ${ }^{[18]}$ and by fibrinogen through NF-kappaB, and ERK and p38 MAPK pathway ${ }^{[19]}$. On the other side, PSCs can be regulated by cytokines in phagocytosis $^{[20]}$ and activation ${ }^{[21]}$.

Since T2DM is a disease characterized by hyperglycemia, therefore, hypergly-cemia may also activate PSCs through inducing production of cytokines IL-1, IL-6, TNF- $\alpha$, in addition to induction of oxdative stress, NF-kappaB, and ERK and 
p38 MAPK pathway ${ }^{[1,5,6]}$. This could be a mechanism of that high glucose aggravates the detrimental effects of pancreatic stellate cells in T2DM.

PSCs activation causes pancreatic fibrosis, which leads to pancreatitis and pancreatic cancers. Studies have conducted to attenuate PSCs activation through inhibiting pancreatic fibrosis. It has been shown that Saikosaponin $d$ ameliorates pancreatic fibrosis by inhibiting autophagy of pancreatic stellate cells via PI3K/ Akt/mTOR pathway ${ }^{[22]}$. PTEN appears to prevent PSCs from further activation and promotes apoptosis through regulation of the AKT and FAK/ERK pathways ${ }^{[23]}$. Resvera-trol inhibits ROS-promoted activation of PSCs via suppression of miR-21 ${ }^{[14]}$. Trans-resveratrol may serve as a therapeutic or an adjuvant agent in anti-fibrotic approaches and/or PSC-relating pathologies [15]. Consistently, in the current study, we found that resveratrol inhibited high glucose induced PSCs proliferation, activation, cytokine expression and secretion in PSCs. Therefore, resveratrol is likely a useful compound that can potentially contribute to ameliorating T2DM, pancreatitis and pancreatic cancer where PSCs is activated by high glucose.

In conclusion, our data supported that resveratrol inhibited high glucose induced PSCs proliferation, activation, cytokine expression and secretion in PSCs. Therefore, resveratrol can be potentially used in therapy of diseases such as T2DM, pancreatitis and pancreatic cancer where PSCs is activated by high glucose.

\author{
Abbreviation \\ a-SMA: Alpha-smooth Muscle Actin \\ Akt: Protein Kinase B \\ ECM: Extracellular Matrix \\ ELISA: Enzyme-linked Immunosorbent \\ assay
}

ERK: Extracellular Regulated Protein Kinases

HG: High Glucose

LG: Low Glucose

LSD: Least Significant Difference

MTT: Methyl Thiazolyl Tetrazolium

mTOR: Mammalian target of rapamycin

MAPK: Mitogen-activated Protein Kinase Pathway

PBST: Phosphate-buffered Saline with Tween 20

PI3K: Phosphatidylinositide 3-kinases

PSCs: Pancreatic Stellate Cells

Res: Resveratrol

ROS: Reactive Oxygen Species

RT-qPCR: Quantitative Reverse Transcrip -tion-polymerase chain reaction

T2DM: Type 2 Diabetes Mellitus

TNF: Tumor Necrosis Factor 


\section{Authors contributions}

Z. Zhou, X.D. Sun. and M. Sang. designed experiments. X.D. Sun, Y. Rao, J.F. An, X.J. Zhou and M. Ssang performed experiments, analyzed data and wrote the manuscript. M.Y. Li, X.S. Gu and X.C. Hao performed some experiments, Z. Zhou,X.D. Sun and M. Sang directed the program. All authors approved the final manuscript revision.

\section{Acknowledgments}

This investigation was supported by the grants from the Natural Science Foundation of Hubei Province of China (2019AHB068, 2017CFB674, 2018CFB701), the Found-ation of Health Commission of Hubei Province (ZY2019F028), the Scientific and Technological Project of Xiangyang City of Hubei Province (to Sang Ming 2017033). This investigation was also supported by the Open Project of Hubei Key Laboratory of Wudang Local Chinese Medicine Research (WDCM2018009), the Innovative Team Project (2017YHKT02) from the Institute of Medicine and Nursing at Hubei University of Medicine..

\section{References}

[1]. Ryu G R, Lee E, Chun H J, et al. Oxidative stress plays a role in high glucose-induced activation of pancreatic stellate cells[J]. Biochemical and biophysical research communications, 2013, 439(2): 258-263.
[2]. Bynigeri R R, Jakkampudi A, Jangala R, et al. Pancreatic stellate cell: Pandora's box for pancreatic disease biology[J]. World journal of gastroenterology, 2017, 23(3): 382.

[3]. Ferdek P E, Jakubowska M A. Biology of pancreatic stellate cells-more than just pancreatic cancer[J]. Pflügers Archiv-European Journal of Physiology, 2017, 469(9): 1039-1050.

[4]. Xue R, Jia K, Wang J, et al. A rising star in pancreatic diseases: pancreatic stellate cells[J]. Frontiers in physiology, 2018, 9: 754.

[5]. Hong $\mathrm{O} \mathrm{K}$, Lee $\mathrm{S} \mathrm{H}$, Rhee $\mathrm{M}$, et al. Hyperglycemia and hyperinsulinemia have additive effects on activation and proliferation of pancreatic stellate cells: Possible explanation of islet-specific fibrosis in type 2 diabetes mellitus[J]. Journal of cellular biochemistry, 2007, 101(3): 665-675.

[6]. Ko S H, Hong O K, Kim J W, et al. High glucose increases extracellular matrix production in pancreatic stellate cells by activating the renin-angiotensin system[J]. Journal of cellular biochemistry, 2006, 98(2): 343-355.

[7]. Nomiyama Y, Tashiro M, Yamaguchi T, et al. High glucose activates rat pancreatic stellate cells through protein kinase $\mathrm{C}$ and p38 mitogen-activated protein kinase pathway[J]. Pancreas, 2007, 34(3): 364-372.

[8]. Zha M, Xu W, Zhai Q, et al. High glucose aggravates the detrimental effects of pancreatic stellate cells on Beta-cell function[J]. International journal of endocrinology, 2014, 2014. 
[9]. Truong V L, Jun M, Jeong W S. Role of resveratrol in regulation of cellular defense systems against oxidative stress[J]. Biofactors, 2018, 44(1): 36-49.

[10]. Wahl D, Bernier M, Simpson S J, et al. Future directions of resveratrol research[J]. Nutrition and healthy aging, 2018, 4(4): 287-290.

[11]. Pannu N, Bhatnagar A. Resveratrol: From enhanced biosynthesis and bioavailability to multitargeting chronic diseases[J]. Biomedicine \& Pharmacotherapy, 2019, 109: 2237-2251.

[12]. Yan B, Cheng L, Jiang Z, et al. Resveratrol inhibits ROS-promoted activation and glycolysis of pancreatic stellate cells via suppression of miR-21[J]. Oxidative medicine and cellular longevity, 2018, 2018.

[13]. Tsang S W, Zhang H, Lin Z, et al. Anti-fibrotic effect of trans-resveratrol on pancreatic stellate cells[J]. Biomedicine \& Pharmacotherapy, 2015, 71: 91-97.

[14]. Kruse, M. L., P. B. Hildebrand, C. Timke, U. R. et al. Isolation, long-term culture, and characterization of rat pancreatic fibroblastoid/stellate cells[J]. Pancreas,2001, 23(1): 49-54.

[15]. Strobel O, Dadabaeva N, Felix K, et al. Isolation and culture of primary human pancreatic stellate cells that reflect the context of their tissue of origin[J]. Langenbeck's archives of surgery, 2016, 401(1): 89-97.
[16]. Zhao W, Ajani J A, Sushovan G, et al. Galectin-3 mediates tumor cell-stroma interactions by activating pancreatic stellate cells to produce cytokines via integrin signaling $[\mathrm{J}]$. Gastroenterology, 2018, 154(5): 1524-1537. e6.

[17]. Masamune A, Kikuta K, Watanabe T, et al. Fibrinogen induces cytokine and collagen production in pancreatic stellate cells $[\mathrm{J}]$. Gut, 2009, 58(4): 550-559.

[18]. Shimizu K, Kobayashi M, Tahara J, et al. Cytokines and peroxisome proliferator-activated receptor $\gamma$ ligand regulate phagocytosis by pancreatic stellate cells[J]. Gastroenterology, 2005, 128(7): 21052118 .

[19]. Mews P, Phillips P, Fahmy R, et al. Pancreatic stellate cells respond to inflammatory cytokines: potential role in chronic pancreatitis[J]. Gut, 2002, 50(4): 535-541.

[20]. Cui L H, Li C X, Zhuo Y Z, et al. Saikosaponin $\mathrm{d}$ ameliorates pancreatic fibrosis by inhibiting autophagy of pancreatic stellate cells via PI3K /Akt/mTOR pathway[J]. Chemico-biological interactions, 2019, 300: 18-26.

[23]. Zhang X, Jin T, Huang X, et al. Effects of the tumor suppressor PTEN on biological behaviors of activated pancreatic stellate cells in pancreatic fibrosis[J]. Experimental cell research, 2018, 373(1-2): 132-144.. 